\title{
PD-1 Blockade Cellular Vesicles for Cancer Immunotherapy
}

\author{
Xudong Zhang, Chao Wang, Jinqiang Wang, Quanyin Hu, Benjamin Langworthy, \\ Yanqi Ye, Wujin Sun, Jing Lin, Tianfu Wang, Jason Fine, Hao Cheng, Gianpietro Dotti, \\ Peng Huang, * and Zhen Gu*
}

Cancer cells resist to the host immune antitumor response via multiple suppressive mechanisms, including the overexpression of PD-L1 that exhausts antigen-specific CD8 ${ }^{+} \mathrm{T}$ cells through PD-1 receptors. Checkpoint blockade antibodies against PD-1 or PD-L1 have shown unprecedented clinical responses. However, limited host response rate underlines the need to develop alternative engineering approaches. Here, engineered cellular nanovesicles (NVs) presenting PD-1 receptors on their membranes, which enhance antitumor responses by disrupting the PD-1/PD-L1 immune inhibitory axis, are reported. PD-1 NVs exhibit a long circulation and can bind to the PD-L1 on melanoma cancer cells. Furthermore, 1-methyl-tryptophan, an inhibitor of indoleamine 2,3-dioxygenase can be loaded into the PD-1 NVs to synergistically disrupt another immune tolerance pathway in the tumor microenvironment. Additionally, PD-1 NVs remarkably increase the density of $\mathrm{CD}^{+}$tumor infiltrating lymphocytes in the tumor margin, which directly drive tumor regression.
However, anti-PD-1 therapy is not effective against all types of cancer and still only a minority of patients benefit from the ICB. ${ }^{[2,4]}$ Meanwhile, most of the available humanized antibodies are produced from mice, which require complicated design and isolation. ${ }^{[5]}$ As a result, the cost of checkpoint antibody therapy remains unaffordable for many patients. ${ }^{[5,6]}$ Therefore, alternative approaches antagonizing the PD-1/PD-L1 inhibitor axis need to be exploited. ${ }^{[7]}$

Natural cell membrane derived vesicles such as exosomes, macrovesicles, and cell membrane extruded vesicles hold great promise for biomedicine. ${ }^{[8]}$ Similarly, bioengineering strategies are promising ways for the enhancement of anticancer immunity. ${ }^{[9]}$ Herein, we engineered cell membrane derived nanovesicles $(\mathrm{NVs})$ to

Cancer immunotherapy aims to leverage the human immune system to eliminate cancer cells. ${ }^{[1]}$ Among them, immune checkpoint blockade (ICB) targeting the programmed death-1 (PD-1)/ programmed death-ligand 1 (PD-L1) pathway induces remarkable clinical responses in various types of cancer, such as melanoma, renal cell carcinoma, nonsmall cell lung cancer, and bladder cancer ${ }^{[2]}$ PD-L1 is the ligand of PD-1 receptor and is upregulated in cancer cells and immune cells to inhibit the effector T cells. ${ }^{[3]}$ Therefore, blocking the interaction between PD-1 and PD-L1 by antibodies boosts the immune response against cancer cells.

Dr. X. D. Zhang, Dr. C. Wang, Dr. J. Q. Wang, Q. Y. Hu, Y. Q. Ye,

Dr.W. J. Sun, Prof. Z. Gu

Joint Department of Biomedical Engineering

University of North Carolina at Chapel Hill and

North Carolina State University

Raleigh, NC 27695, USA

E-mail: zgu@email.unc.edu

Dr. X. D. Zhang, Prof. J. Lin, Prof. T. F. Wang, Prof. P. Huang Guangdong Key Laboratory for Biomedical Measurements and

Ultrasound Imaging

School of Biomedical Engineering

Health Science Center

Shenzhen University

Shenzhen 518060, China

E-mail: peng.huang@szu.edu.cn

Dr. B. Langworthy, Prof. J. Fine

Department of Biostatistics

University of North Carolina at Chapel Hill

Chapel Hill, NC 27599, USA

DOI: 10.1002/adma.201707112 display PD-1 receptors, which enhance the cancer immunotherapy through disrupting the PD-1/PD-L1 immune inhibitory axis (Figure 1a). The PD-1 NVs can bind to the surface of tumor cells and achieve PD-L1 blockade (Figure 1a-c). This blockade is expected to effectively revert the exhausted tumor antigenspecific $\mathrm{CD}^{+} \mathrm{T}$ cells to attack the tumor cells. In addition, the NVs can also serve as carriers for other therapeutics to perform combination delivery. Indoleamine 2,3-dioxygenase (IDO) is an immunosuppressive molecule overexpressed by tumor and $\mathrm{DC}$ cells $\left(\mathrm{IDO}^{+} \mathrm{DCs}\right)$ to limit the proliferation and function of

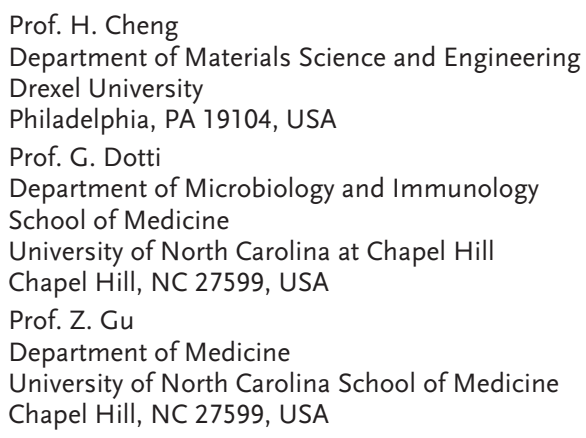

Prof. H. Cheng

Drexel University

Philadelphia, PA 19104, USA

Prof. G. Dotti

School of Medicine

University of North Carolina at Chapel Hill

Chapel Hill, NC 27599, USA

Department of Medicine

Chapel Hill, NC 27599, USA 


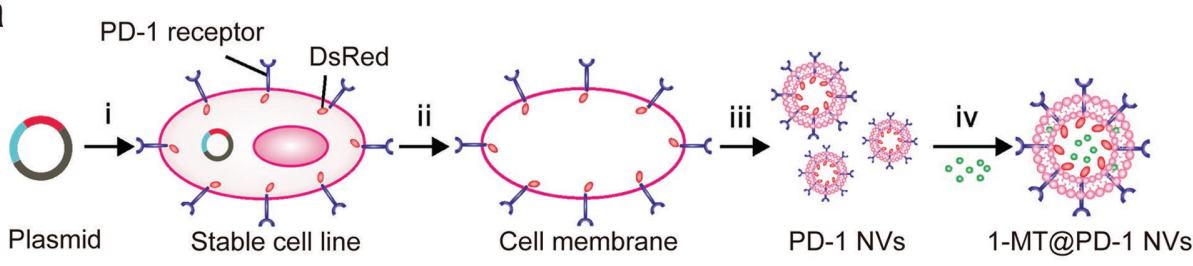

b
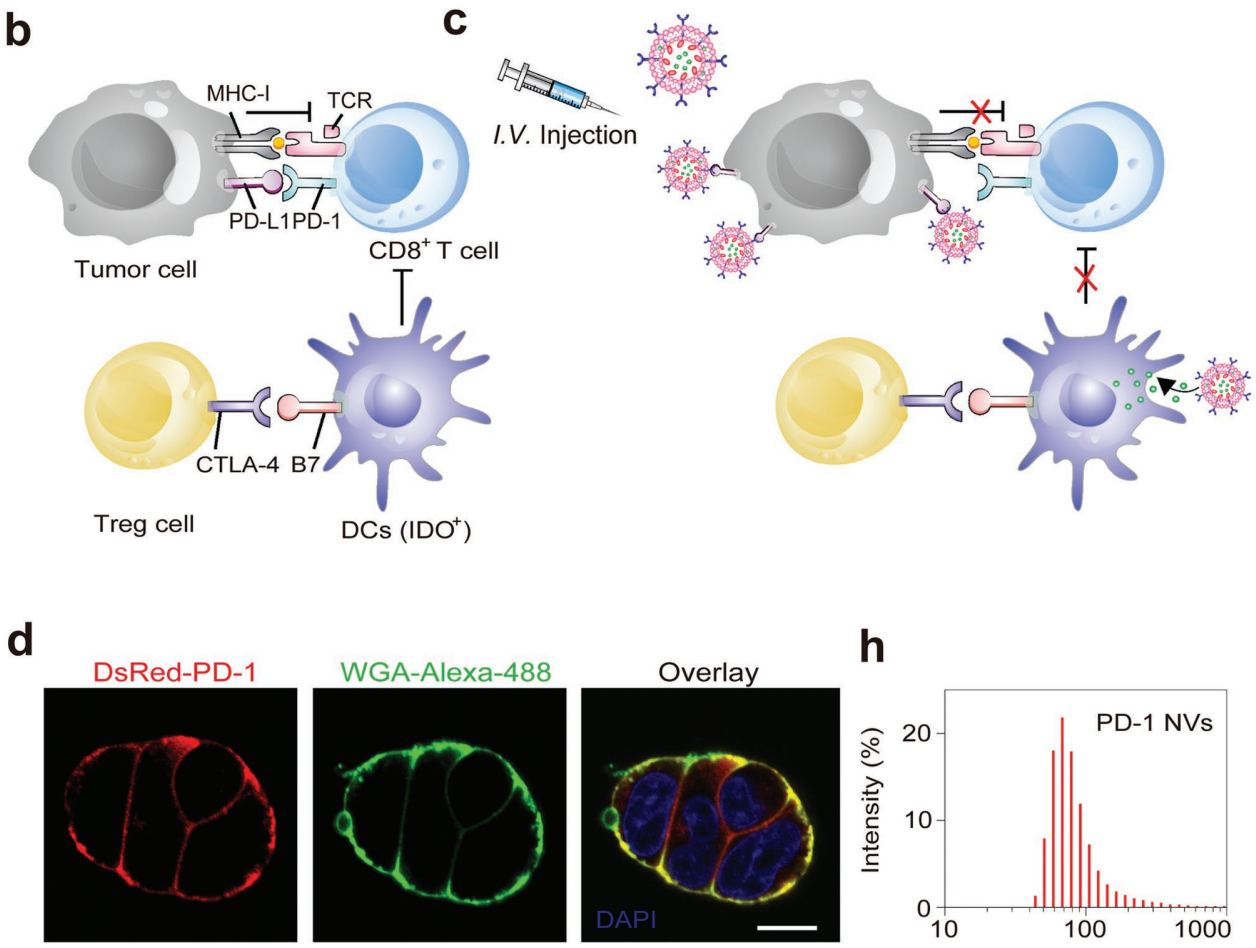

e
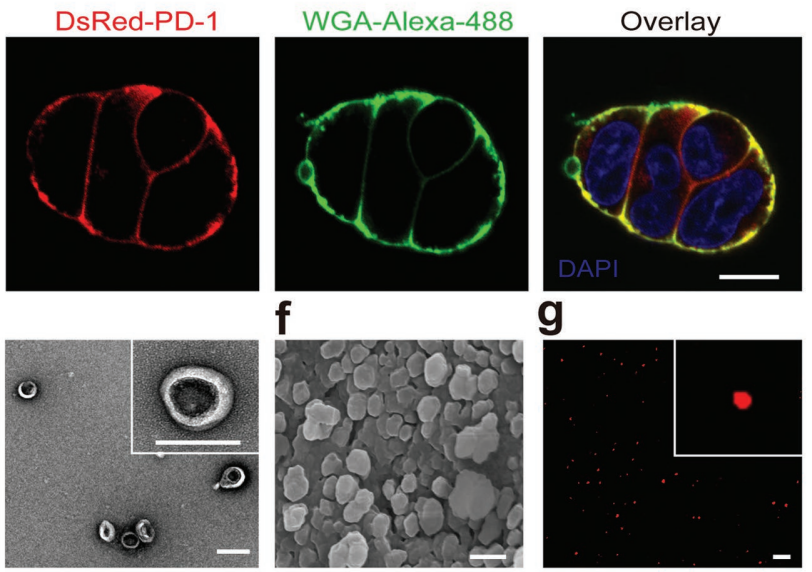

h
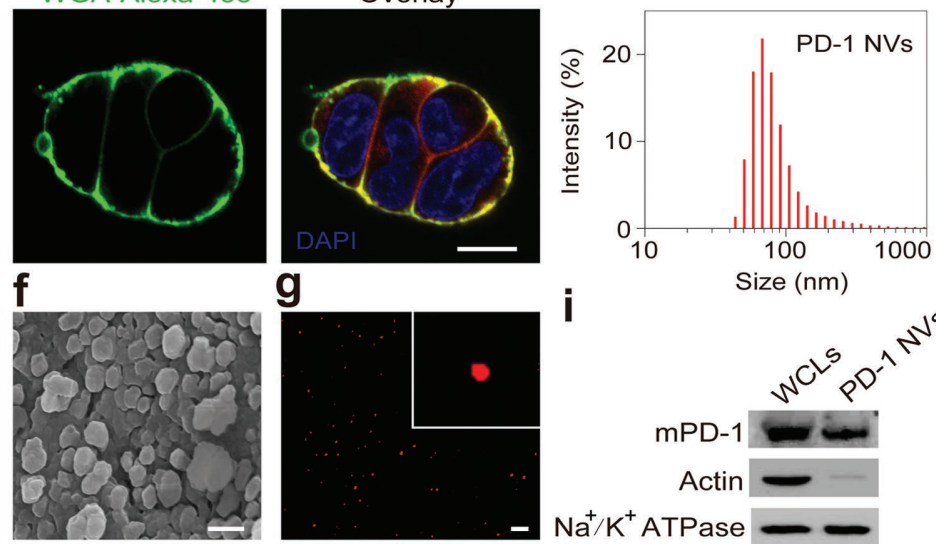

i

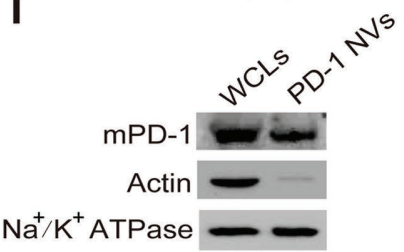

Figure 1. Schematic illustration and characterization of PD-1 blockade cellular NVs for cancer immunotherapy. a) Schematic illustration shows the praparation of PD-1 NVs loaded with 1-MT. i) Engineering of HEK 293T cell line stably expressing mouse PD-1 receptors on the cell membranes. ii) Harvesting of the cell membrane expressing PD-1 receptors. iii) Preparation of PD-1 NVs through extrusion. iv) Loading 1-MT into PD-1 NVs. b) PD-L1 exhausts CD8 ${ }^{+} T$ cells by interacting with PD-1 receptors. The expression of IDO is induced by Treg cells, which inhibits the activity of CD8 ${ }^{+} \mathrm{T}$ cells. MHC-I: major histocompatibility complex class I; TCR: T cell receptor; CTLA4: cytotoxic T-lymphocyte associated protein 4. c) PD-L1 blockade by PD-1 $\mathrm{NVs}$ reverts the exhausted CD8 $8^{+}$cells to attack tumor cells. The release of IDO inhibitor 1-MT also reverts the exhausted CD $8^{+} \mathrm{T}$ cells. d) Establishment of HEK 293T cell line stably expressing mouse PD-1 on cell membranes. WGA Alexa-Fluor 488 dye was used to label cell membrane. Scale bar: $10 \mu$ m. e) The TEM image showed the shape and size of PD-1 NVs. Scale bar: $100 \mathrm{~nm}$. f) Cryoscanning electron microscopy (CSEM) image showed the natural shape of the PD-1 NVs (Scale bar: $100 \mathrm{~nm}$ ). g) The confocal image indicated the existence of DsRed-PD-1 NVs by the red spots. Scale bar: $1 \mu \mathrm{m}$. $\mathrm{h}$ ) The size distribution of PD-1 NVs measured by dynamic light scattering (DLS) analysis. i) Western blot assay exhibited the expression of mouse PD-1 (mPD-1) receptors on the NVs and whole cell lysate (WCLs) of the stable cell line. $\mathrm{Na}^{+} \mathrm{K}^{+}$ATPase was used as a loading control.

effector T cells. ${ }^{[10]}$ Here we encapsulated 1-methyl-tryptophan (1-MT), a small molecule inhibitor of IDO into PD-1 NVs to simultaneously block the PD-1/PD-L1 axis and overcome the inhibitory effects of IDO on effector T cells within the tumor microenvironment (TME) (Figure 1c). ${ }^{[11]}$

To prepare PD-1 NVs, we established HEK 293T cells that stably express the mouse PD-1 receptor on the cell membrane. HEK 293T cell line has been widely used in cell biology research and biotechnology industry because it can be robustly transfected and produces high amount of recombinant proteins. ${ }^{[12]}$ DsRed protein-tag was included in the C-terminal portion of PD-1 receptor protein, which made the protein-tag close to the inner leaflet of cell membranes, while the functional domain of the receptors is extracellular (Figure 1a). Therefore, we cloned the mouse PD-1 receptor cDNA into a mammalian expression vector. The transfected HEK 293T cells were selected 
with hygromycin B to establish a stable cell line. Notably, the death receptor PD-1 was mainly expressed and localized on the cell membranes (Figure 1d). Under the selection pressure of hygromycin B, the cell line continued to express DsRed PD-1 receptors for more than twenty passages (Figure S1, Supporting Information). Furthermore, we labeled the cell membranes with Alexa-Fluor 488 conjugated wheat germ agglutinin (WGA) to confirm the localization of the PD-1 receptors. As expected, the red fluorescence of $\mathrm{D}$ sRed p rotein c olocalized with g reen fluorescence of WGA Alexa-Fluor 488 dye on the cell membranes (Figure 1d).

Next, the engineered HEK 293T cells were cultured and lyzed to isolate the cell membranes. The cell membrane vesicles expressing PD-1 receptors were prepared by a serial extrusion of vesicles through 0.8 and $0.22 \mu \mathrm{m}$ pore-sized polycarbonate membrane filters. ${ }^{[13]}$ A fter extrusion through the $0.8 \mu \mathrm{m}$ p ore-sized polycarbonate membrane filters, major cell membrane vesicles (MVs) were obtained. The red-light spots in the confocal image demonstrated the existence of DsRed-PD-1 on MVs (Figure S2a, Supporting Information). The size distribution of MVs was measured by the dynamic light scattering (DLS) analysis (Figure S2b, Supporting Information). The MVs were then extruded through $0.22 \mu \mathrm{m}$ pore-sized polycarbonate membrane filters. The $\mathrm{h}$ arvested NVs were further purified by a density gradient ultracentrifugation. ${ }^{[13]}$ Next, we characterized the morphology of the NVs by electron microscopy. The negatively stained NVs revealed that they were closed vesicles using transmission electron microscopy (TEM) (Figure 1e). The NVs were also scanned by the cryoscanning electron microscopy (CSEM), which showed that the NVs had a spherical shape (Figure 1f). The zeta potential of the $\mathrm{NVs}$ was determined as $-10 \mathrm{mV}$ (Figure S2c, Supporting Information). Moreover, the expression of PD-1 receptors on the NVs was detected using confocal imaging and western blot. The confocal image exhibited red-colored spots indicating the existence of DsRed-PD-1 NVs (Figure 1g). DLS analysis showed that the average diameter of NVs was around 90-100 nm (Figure 1h). Additionally, western blot analysis indicated that the purified N Vs d isplay the P D-1 r eceptors (Figure $1 \mathrm{i}$ ). To v erify that whether the PD-1 receptors maintained an outside-out orientation on NV surfaces, we performed an immunoprecipitation assay (IP). It was demonstrated that the PD-1 antibody pulled down the majority of PD-1 NVs, which demonstrated that PD-1 receptors have a correct outside-out orientation on the most PD-1 NVs (Figure S3, Supporting Information).

Cancer cells exhaust antigen-specific $\mathrm{CD}^{+} \mathrm{T}$ cells through overexpression of PD-L1 ligands that interact with PD-1 receptors. ${ }^{[2 a]}$ To investigate whether PD-1 NVs bind to melanoma cells, we incubated the PD-1 NVs with B16F10 melanoma cells in vitro. DsRed proteins fused with PD-1 receptors provided red fluorescence, which was used as a fluorescent signal label of the PD-1 NVs. WGA Alexa-Fluor 488 dye was used to stain the cell membranes of the B16F10 melanoma cells. Remarkably, we observed that PD-1 NVs effectively bound around the cell membrane surface of B16F10 cells after incubation for $2 \mathrm{~h}$ (Figure 2a). In contrast, Cy5.5 labeled the free NVs had low membrane binding affinity (Figure 2a). In addition, we also detected the interaction between PD-1 NVs and dendritic cells (DCs). PD-1 NVs were incubated with bone marrowderived DCs (BMDCs) for $2 \mathrm{~h}$. The confocal image showed that
DsRed-PD-1 NVs could effectively bind and be internalized by the BMDCs after $2 \mathrm{~h}$ (Figure $2 \mathrm{~b}$ ). To investigate whether the binding of PD-1 NVs on the B16F10 cells was through the interaction between PD-1 and PD-L1, we first detect the colocalization between PD-1 receptors on NVs and PD-L1 on B16F10 cells. PD-1 NVs were incubated with EGFP-PD-L1 expressing B16F10 cells for $5 \mathrm{~h}$. Notably, PD-1 NVs were colocalized with EGFP-PD-L1 on the B16F10 melanoma cells (Figure 2c). To confirm the molecular binding between $\mathrm{PD}-1$ receptors on NVs and PD-L1 on the B16F10 cells, we added aPD-L1 antibody to block the PD-L1 on the B16F10 cells. The confocal images showed that PD-1 NVs binding was dramatically reduced when PD-L1 antibody (aPD-L1) was preincubated with the cells (Figure S4a, Supporting Information). Moreover, the flow cytometric data also showed that the quantity of PD-1 NVs binding with B16F10 cells is significantly reduced when PD-L1 antibody was preincubated with the cells (Figure 2d; Figure S4b, Supporting Information). We also employed coimmunoprecipitation (CO-IP) assay to detect the molecular interaction between PD-1 receptor and PD-L1. After incubation of the PD-1 NVs with B16F10 melanoma cells for $20 \mathrm{~h}$, the cells were harvested. PD-1 primary antibody was used to pull down the PD-1 receptors on the NVs. Remarkably, PD-L1 was pulled down together with PD-1 receptors by the PD-1 antibody (Figure 2e), indicating that PD-1 NVs physically interact with PD-L1 expressed by B16F10 cells. Together, these results substantiated that the NVs presenting PD-1 on the surface could effectively interact with tumor cells through the binding between PD-1 receptor and PD-L1.

To investigate the systemic biodistribution and kinetics of PD-1 NVs, we labeled the free NVs and PD-1 NVs with Cy5.5. Free NVs and PD-1 NVs were injected into the mice through tail-vein. As shown in Figure 2f, the PD-1 NVs had higher blood retention compared to the free NVs. The PD-1 NVs exhibited $32 \%$ and $17 \%$ overall retention compared to $12 \%$ and $1.7 \%$ retention of the free NVs at 8 and 48 h, respectively. Next, we examined the in vivo tissue distribution of PD-1 NVs. B16F10tumor-bearing mice received Cy5.5-labeled PD-1 NVs via tailvein injection. Notably, we observed the accumulation of Cy5.5 fluorescence of PD-1 NVs primarily at the liver, kidney, and tumor sites (Figure $2 \mathrm{~g}, \mathrm{~h}$ ). To further assess the biodistribution of the PD-1 NVs, we quantified the Cy5.5-labeled NVs in the sections of organs and tumors by confocal imaging. The WGA Alexa-Fluor 488 dye was used to stain the cell membrane in the tissue sections. The distribution of the PD-1 NVs paralleled the imaging data showing intensive accumulation of the PD-1 NVs in the tumor tissue sections (Figure 2i).

To determine whether the PD-1 NVs promote the mice immune response to the melanoma tumor, we established a melanoma tumor model in which B16F10-luc cells were inoculated subcutaneously in C57BL/6 mice. Five days after tumor inoculation, $25 \mathrm{mg}$ (based on protein weight) per $\mathrm{kg}$ free NVs and 20-30 mg per kg PD-1 NVs were inoculated in mice through tail-vein injection. Tumor growth was monitored by measuring both bioluminescence signals and tumor size. Notably, the growth of B16F10 tumors was significantly delayed in mice treated with PD-1 NVs at the dosage of 20, 25, and $30 \mathrm{mg} \mathrm{kg}^{-1}$ (Figure S5, Supporting Information). To confirm the in vivo antitumor effect of PD-1 NVs, we employed 
a

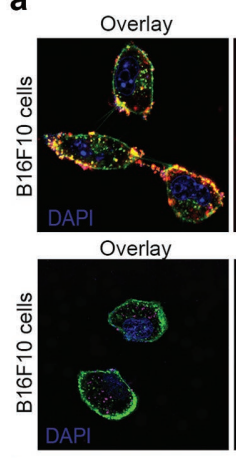

b

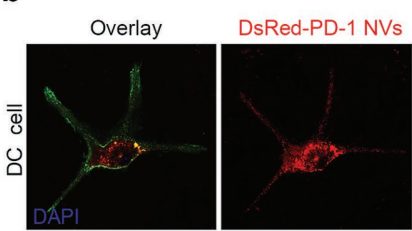

f
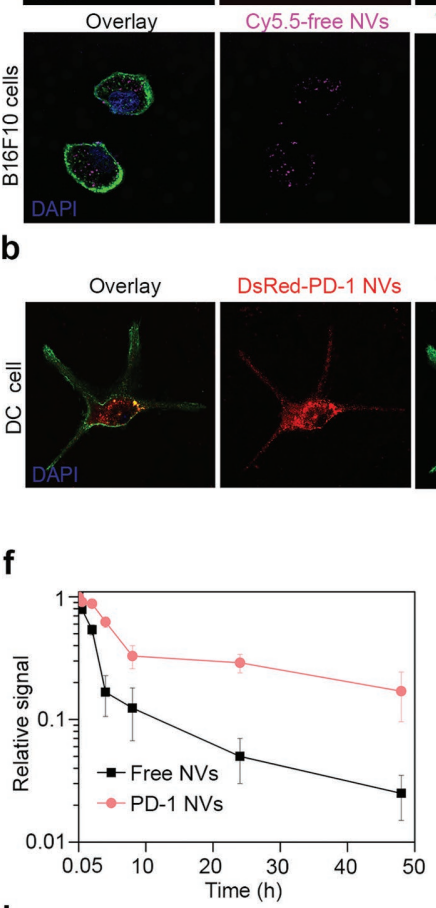

C
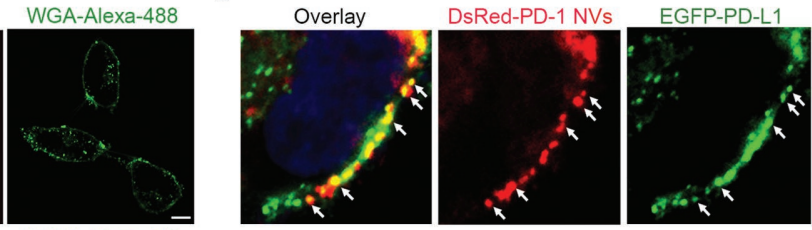

WGA-Alexa-488
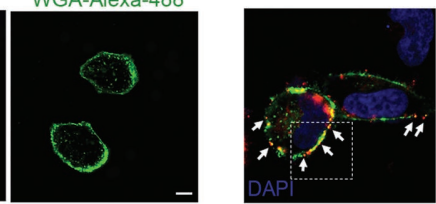

d
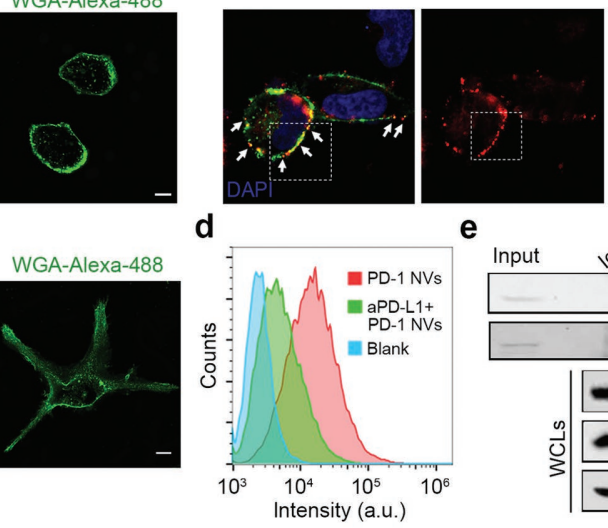

e

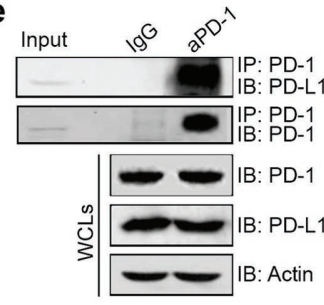

g

h

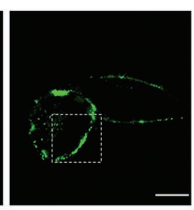

Free NVs PD-1 NVs
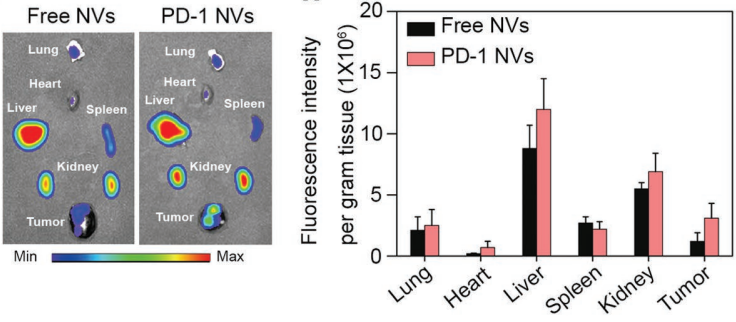

WGA-Alexa-488/PD1-NVs/ DAP
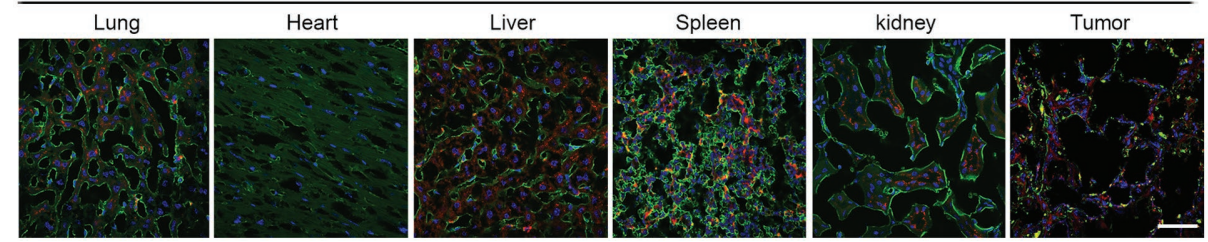

Figure 2. In vitro biological behavior and in vivo biodistribution of PD-1 NVs. a) DsRed-PD-1 NVs bound on the cell membrane of B16F10 cancer cells. PD-1 NVs $\left(50 \mu \mathrm{g} \mathrm{mL}^{-1}\right.$, protein weight) or PD-1 free NVs labeled with Cy5.5 $\left(50 \mu \mathrm{g} \mathrm{mL}^{-1}\right.$, protein weight) were incubated with B16F10 cells for $2 \mathrm{~h}$. WGA Alexa-Fluor 488 dye was used to detect B16F10 cell membrane (Scar bar: $10 \mu \mathrm{m})$. b) DsRed-PD-1 NVs were internalized by DCs. PD-1 NVs (50 $\mu$ g mL ${ }^{-1}$ ) were incubated with DCs for $2 \mathrm{~h}$. WGA Alexa-Fluor 488 dye was used to detect DC membrane. Scar bar: $10 \mu \mathrm{m}$. c) B16F10 cells were transfected with EGFP-PD-L1 plasmid for $20 \mathrm{~h}$, then incubated with PD-1 NVs $\left(50 \mu \mathrm{g} \mathrm{mL} \mathrm{L}^{-1}\right)$ for $5 \mathrm{~h}$; the colocalization of PD-1 NVs and PD-L1 proteins were detected (Scar bar: $10 \mu \mathrm{m})$. The above images are the enlarged ones in the white collar on the underside images. d) The representative flow cytometric analysis images of PD-1 NVs binding with B16F10 cells (gated on DsRed ${ }^{+}$). PD-1 NVs $\left(50 \mu \mathrm{g} \mathrm{mL}^{-1}\right)$ were incubated with B16F10 cells for $5 \mathrm{~h}$. Or aPD-L1 antibody $\left(20 \mu \mathrm{g} \mathrm{mL} \mathrm{L}^{-1}\right.$ ) was incubated with the cells for $4 \mathrm{~h}$ before the PD-1 NVs were added in the culture medium as indicated. e) CO-IP and western blot were used to examine the interaction between PD-1 (on NVs) and PD-L1 (on B16F10 cells). Immunoprecipitation (IP); Immunoblot (IB). f) Cy5.5 labeled free NVs and PD-1 NVs were injected through tail-vein of the mice. Fluorescence was measured at different time points as indicated $(n=3)$. Error bar, mean \pm s.d. g) The IVIS spectrum images of distribution offree NVs and PD-1 NVs in tumorand majororgans. h) Fluorescence intensity pergram oftissue in tumorand major organs as indicated $(n=3)$. Error bar, mean \pm s.d. i) The distribution of PD-1 NVs in the organs and tumor sections was detected using confocal microscope. Scar bar: $100 \mu \mathrm{m}$.

treatment with the administration of the anti-PD-L1 antibody as a positive control. The mice were divided into three groups: $25 \mathrm{mg}$ per $\mathrm{kg}$ free NVs (Group 1) and PD-1 NVs (Group 2) were injected in mice through the tail vein every three days for five cycles. Anti-PD-L1 antibody (aPD-L1, Group 3) was also injected into mice at $2 \mathrm{mg} \mathrm{kg}^{-1}$ as a positive control group. Tumor growth was monitored using both bioluminescence signals and tumor size. Of note, PD-1 NVs significantly delay the B16F10 melanoma tumor growth, comparable to the treatment with aPD-L1 (Figure 3a-c). Consequently, PD-1 NVs improved the survival of the mice (Figure 3d), and $20 \%$ of mice survived more than $60 \mathrm{~d}$ upon PD-1 NVs treatment. Moreover, there was no obvious weight loss during the treatment (Figure 3e). No significant antitumor effects were observed in the mice treated with free NVs.

Exhausted $\mathrm{CD}^{+} \mathrm{T}$ cells express inhibitory receptor proteins, including PD-1, TIGIT, LAG3, and TIM3, ${ }^{[14]}$ and have reduced capacity to produce immune cytokines, such as IFN- $\gamma$ 
a

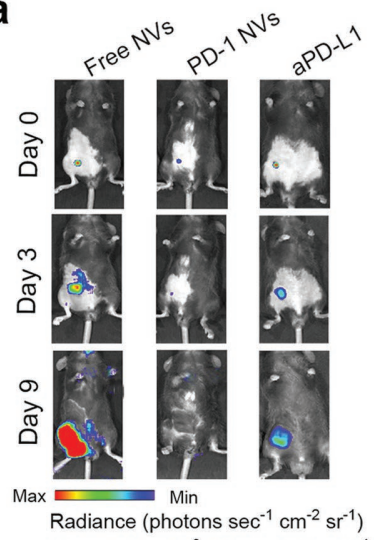

$\operatorname{Min}=2.44 \times 10^{3} \operatorname{Max}=2.44 \times 10^{4}$

C

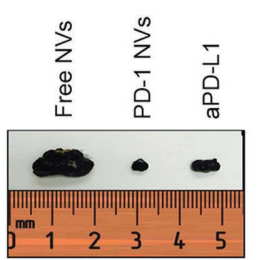

g
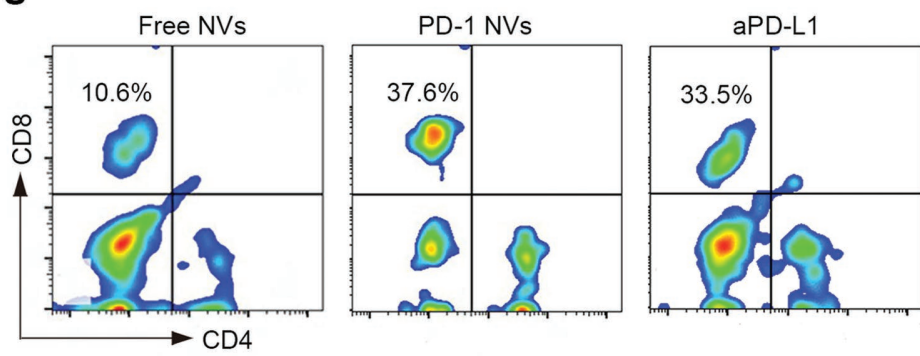

i

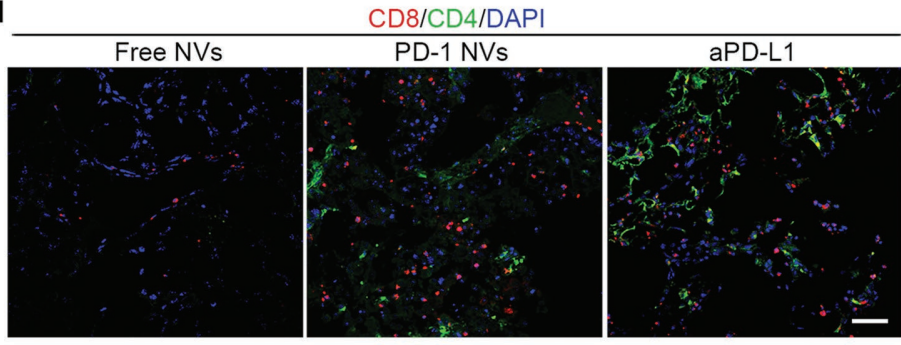

d

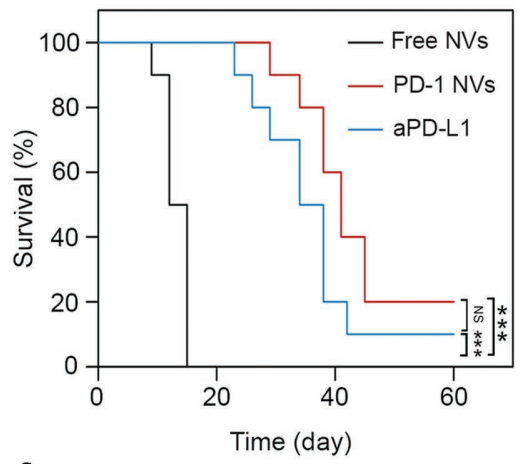

f
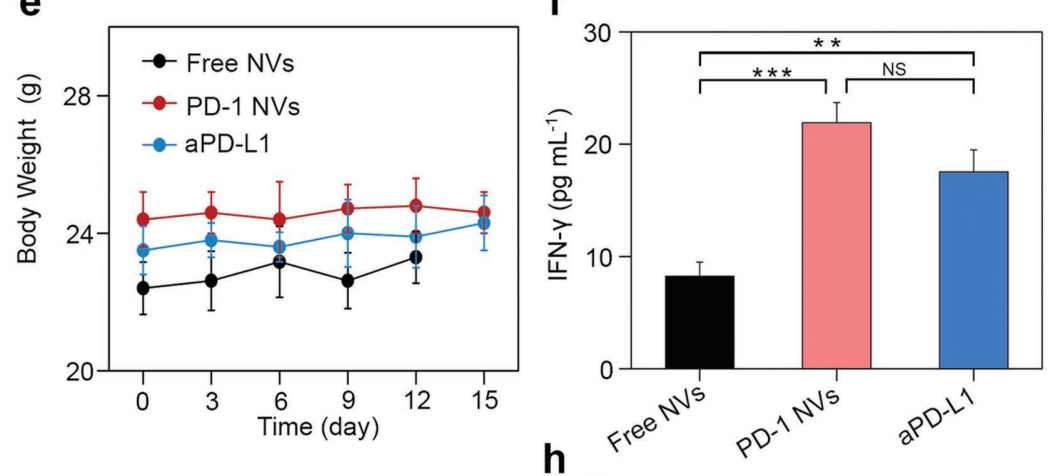

h
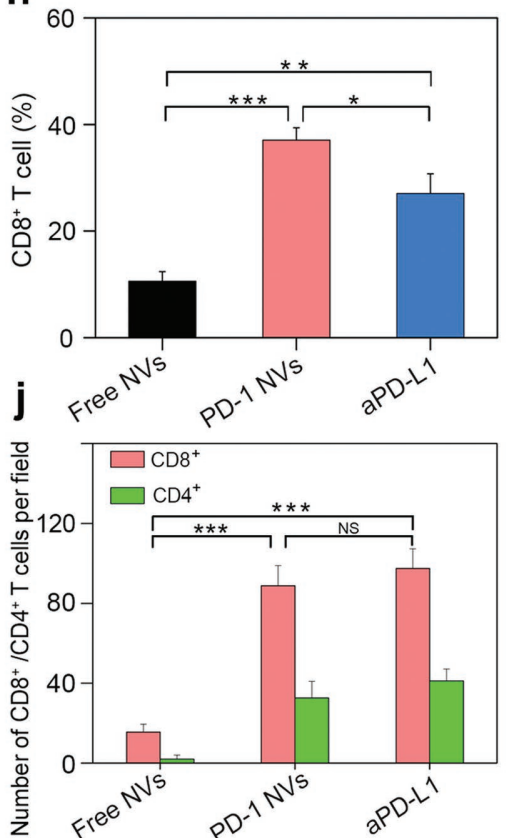

Figure 3. In vivo antitumor effect of PD-1 NVs. a) In vivo bioluminescence imaging of the B16F10 melanoma tumor of different mice groups at different time points after the tail-vein injection of free NVs, PD-1 NVs and PD-L1 antibody. Day 0: the day for the first time of treatment. b) Average tumor volumes of the treated mice in different groups $(n=7)$. Error bar, mean \pm s.e.m. c) Image of representative tumor extracted from euthanized mice of each group. d) Survival curves for the mice received the treatment of PD-1 NVs, PD-L1 antibody, and free NVs $(n=10)$. e) Body weights of mice received the treatment and control mice. Error bar, mean \pm s.d. f) IFN- $\gamma$ levels in serum from mice isolated at day 20 after mice received the first indicated treatment $(n=3)$. Error bar, mean \pm s.d. g,h) Representative plots (g) and quantitative analysis (h) of T cells (gated on CD3 ${ }^{+}$cells) in treated tumor analyzed by flow cytometry $(n=3)$. Error bar, mean \pm s.d. i) Representative image and j) quantitative analysis of immunofluorescence staining of the tumor sections showing infiltrated $C D 4^{+} T$ cells and $C D 8^{+} T$ cells $(n=3)$. Error bar, mean $\pm \mathrm{s}$.d. Scar bar: $100 \mu \mathrm{m}$. Throughout, NS: no significant, $* P<0.05, * * P<0.01$, $* * * P<0.001$; by one-way analysis of variance (ANOVA) with b,f,h,j) Tukey post-hoc tests or by d) Log-Rank (Mantel-Cox) test. 
and TNF- $\alpha{ }^{[15]}$ To assess whether PD-1 NVs treatment reduces $\mathrm{T}$ cell exhaustion and maintain their antitumor function, we measured IFN- $\gamma$ and TNF- $\alpha$ levels in the serum of the treated mice by the end of the fifth cycles. IFN- $\gamma$ levels in the serum of mice treated with either PD-1 NVs or aPD-L1 were significantly increased (Figure 3f), while TNF- $\alpha$ levels remained unchanged (Figure S6, Supporting Information). The infiltration of $\mathrm{CD}^{+} \mathrm{T}$ cells in the harvested tumor was analyzed by flow cytometry. The percentage and number of activated CD ${ }^{+}$ $\mathrm{T}$ cells were significantly increased in tumor collected from mice treated with either PD-1 NVs or aPD-L1 groups as compared to control group (Figure 3g, h). Similarly, higher densities of $\mathrm{CD}^{+} \mathrm{T}$ cells were detected by immunofluorescence in tumors collected from mice treated with either PD-1 NVs or aPD-L1 (Figure 3i,j). Finally, after five cycles of treatments, blood cell counts showed that lymphocyte and monocyte contents slightly decreased in mice treated with PD-1 NVs, while the lymphocyte ratios were not affected (Figure S7a,b, Supporting Information). Additionally, the plasma level of immunoglobulin E (IgE) antibody, produced by the immune system overreacts to an allergen, ${ }^{[16]}$ did not significantly increase after five cycles of the treatment with PD-1 NVs (Figure S8, Supporting Information).

Next, we loaded the IDO inhibitor 1-MT into the PD-1 NVs to investigate the combinatorial therapy of IDO inhibitor and immune checkpoint blockage. High loading capacity $(\approx 24 \%$, drug/protein weight ratio) of 1-MT was achieved by employing the electric shock method compared to the traditional incubation methods $(\approx 16 \%)$ (Figure S9a, Supporting Information). The release of 1- MT from the PD-1 NVs was also tested. 1-MT can be rapidly released from the NVs within $24 \mathrm{~h}$ in vitro (Figure S9b, Supporting Information). Furthermore, to determine the inhibitory effect of 1-MT released by 1-MT-loaded PD-1 NVs, we performed an IDO inhjibition assay using HeLa cells that express IDO after IFN- $\gamma$ stimulation. Remarkably, PD-1 NVs loaded with 1-MT had better inhibitory effect compared to the free 1-MT and 1-MT-loaded free NVs (Figure S10, Supporting Information).

To demonstrate whether the simultaneous IDO inhibition and PD-L1 blockade provided by 1-MT-loaded PD-1 NVs
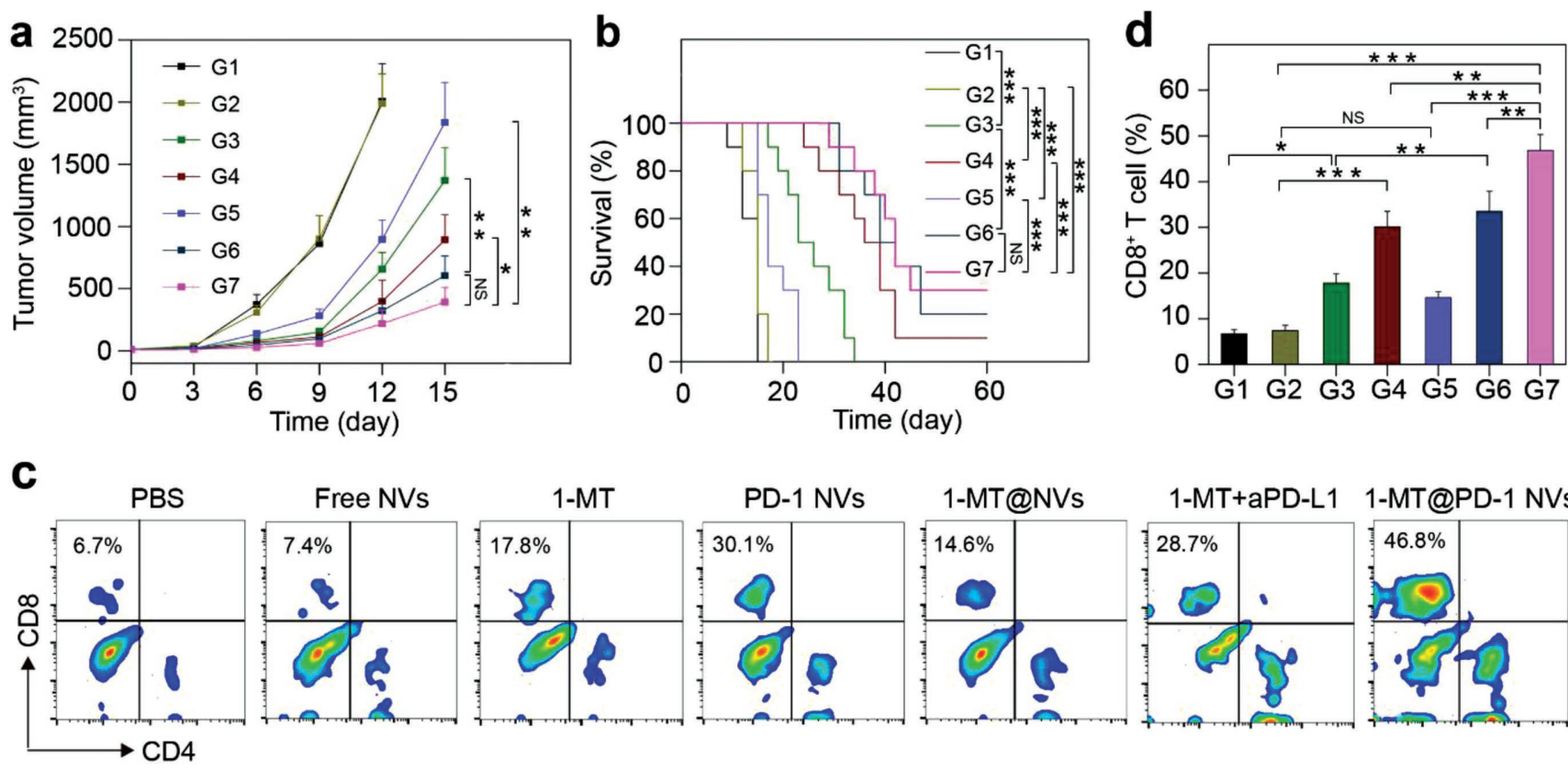

1-MT+aPD-L1 1-MT@PD-1 NVs

$\mathbf{e}$ CD8/CD4/DAPI

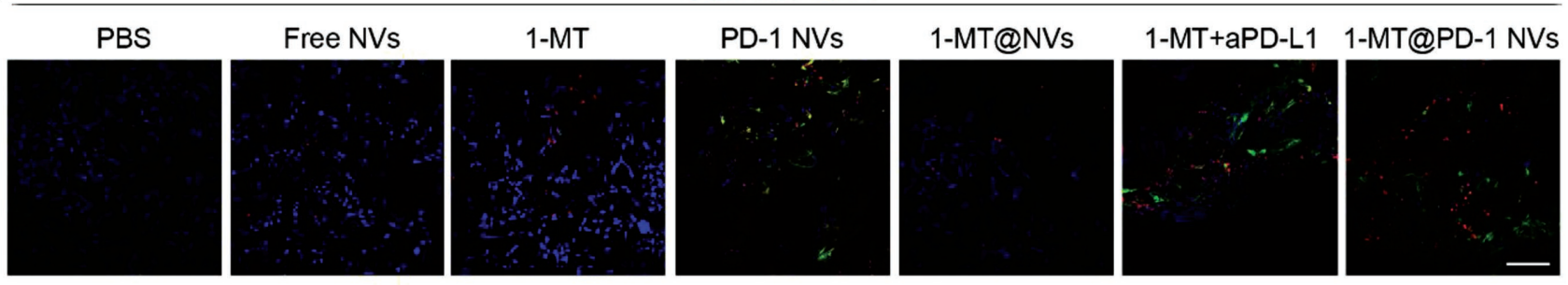

Figure 4. In vivo suppression of tumor growth by 1-MT-loaded PD-1 NVs. a) Average tumor volumes of the treated mice in different groups as indicated $(n=7)$. Error bar, mean \pm s.e.m. b) Survival curves for the mice received different treatment as indicated $(n=10)$. c) Representative flow cytometry plots and d) quantitative analysis of T cells in the tumors from different treatment groups $(n=3)$. The cells were pregated for positive CD $3^{+}$expression. Error bar, mean \pm s.d. e) Immunofluorescence of the tumors showed infiltrated CD4 ${ }^{+} \mathrm{T}$ cells and CD8 ${ }^{+} \mathrm{T}$ cells. Scar bar: $100 \mu \mathrm{m}$. Throughout, NS: no significant, $* P<0.05, * * P<0.01, * * * P<0.001$; a,d) two two-way ANOVA analyses were carried out to do the analyses. First two-way ANOVA with Tukey post-hoc test analysis carried out between the group of free-NVs (G2), PD-1 NVs (G4), 1-MT@NVs (G5), and 1-MT@PD1- NVs (G7). The two factors considered were 1-MT and PD-1. The second two-way ANOVA with Tukey post-hoc test carried out between the groups of the PBS control (G1), aPD-L1, 1-MT (G3), and aPD-L1+1-MT (G6). The two factors in this model were 1-MT and aPD-L1 or b) by Log-Rank (Mantel-Cox) test. 
enhance antitumor activity, B16F10-luc tumor bearing mice were treated with PBS (Group 1), free NVs (Group 2), free 1-MT (Group 3), PD-1 NVs (Group 4), 1-MT-loaded free NVs (Group 5), 1-MT plus aPD-L1 (Group 6) and 1-MT-loaded PD-1 NVs (Group 7) every $3 \mathrm{~d}$ for five cycles. Tumor growth was monitored by measuring both bioluminescence signals and sizes of the tumors. We found high response rate in the mice treated with free 1-MT and 1-MT-loaded free NVs, however, limited suppression of tumor growth was observed (Figure 4a; Figures S11 and S12, Supporting Information). This nonideal efficacy may be because multiple immune suppression mechanisms exist within the TME. Notably, PD-1 NVs had better antitumor effects as compared to 1-MT (Figure 4a; Figures S11 and S12, Supporting Information). The mice treated with 1-MT plus aPD-L1 exhibited significantly delayed progress of the melanoma tumors (Figure 4a; Figures S11 and S12, Supporting Information). Importantly, treatment with 1-MT-loaded PD-1 NVs showed a high response rate to the melanoma tumor, which was much more efficient than the treatment with 1-MT or PD-1 NVs alone (Figure 4a; Figures S11 and S12, Supporting Information), and are comparable to the treatment with 1-MT plus aPD-L1 (Figure 4a; Figures S11 and S12, Supporting Information). Furthermore, the dual inhibition of IDO and PD-L1 by 1-MT-loaded PD-1 NVs improved the survival of the treated mice without obvious weight loss (Figure 4b; Figure S13, Supporting Information). We further examined the density of the $\mathrm{CD} 8^{+} \mathrm{T}$ cells in the tumor margin of different treatment groups. Tumor-infiltrated $\mathrm{CD}^{+} \mathrm{T}$ cells from tumors in all the treatment groups were harvested and analyzed by flow cytometry and immunofluorescence. It was demonstrated that treatment with free 1-MT and 1-MT-loaded $\mathrm{NVs}$ increased the number of infiltrating $\mathrm{CD}^{+} \mathrm{T}$ cells by $\approx 15-20 \%$ compared to the PBS-treated group (Figure $4 c, d$ ). Immunofluorescence staining confirmed that PD-1 and 1-MTloaded PD-1 NVs significantly enhanced the density of tumorinfiltrated $\mathrm{CD}^{+} \mathrm{T}$ cells (Figure 4e). The therapeutic efficacy of combination treatment was better than the individual ones. Infiltration of $\mathrm{CD}^{+} \mathrm{FoxP}^{+} \mathrm{T}$ cells was also studied. Notably, $\mathrm{CD}^{+}{ }^{+} \mathrm{FoxP}^{+} \mathrm{T}$ cells were reduced in 1-MT-loaded PD-1 NVs group as well compared to control group (Figure S14, Supporting Information). Finally, major organs such as liver, spleen, kidney, heart, and lung were collected and assessed by immunohistochemistry without showing any obvious sign of organ damage (Figure S15, Supporting Information). These data revealed that IDO inhibition combined with PD-L1 blockage PD-1 NVs significantly disrupted the immunosuppression of TME, which enhanced the elimination of cancer cells by the host's immune system.

In summary, we engineered cellular nanocarriers displaying PD-1 receptors that effectively bind to PD-L1 on the tumor cells and disrupt the PD-1/PD-L1 inhibitory axis. PD-L1 blockade by PD-1 NVs significantly enhanced the immune response against the melanoma tumor in vivo. Furthermore, PD-1 NVs could also be adapted to carry a variety of therapeutics to achieve a synergistic efficacy. IDO inhibition and PD-L1 blockade were achieved by 1-MT-loaded PD-1 NVs. The disruption of dual immune tolerance mechanisms in tumors remarkably suppressed the melanoma tumor growth in vivo. Thus, PD-L1 blockade by PD-1 cellular NVs provides a promising strategy that leverages functions of both delivery vehicles and encapsulated drugs for enhancing immunotherapy. ${ }^{[17]}$

\section{Supporting Information}

Supporting Information is available from the Wiley Online Library or from the author.

\section{Acknowledgements}

This work was supported by grants from the Alfred P. Sloan Foundation (Sloan Research Fellowship), NC TraCS, the National Institutes of Health (Clinical and Translational Science Award (CTSA, NIH grant 1L1TR001111), a pilot grant from the University of North Carolina (UNC) Cancer Center, the National Natural Science Foundation of China (31771036, 51703132, 51573096, 81401465), the Basic Research Program of Shenzhen (JCYJ20170412111100742, JCYJ20160422091238319), Fok Ying-Tong Education Foundation for Young Teachers in the Higher Education Institutions of China (161032), and China Postdoctoral Science Foundation (2017M612742). The authors acknowledge L. Huang at UNC at Chapel Hill for providing the B16F10-Luc-GFP. Female C57BL/6 mice were purchased from Jackson Lab (USA). Mouse studies were performed in the context of an animal protocol approved by the Institutional Animal Care and Use Committee at North Carolina State University and University of North Carolina at Chapel Hill.

\section{Conflict of Interest}

The authors declare no conflict of interest.

\section{Keywords}

cancer immunotherapy, cell therapy, drug delivery, immune checkpoint blockade, nanomedicine

Received: December 5, 2017

Revised: January 20, 2018

Published online: April 14, 2018

[1] a) P. Sharma, J. P. Allison, Cell 2015, 161, 205; b) A. K. Palucka, L. M. Coussens, Cell 2016, 164, 1233; c) I. Mellman, G. Coukos, G. Dranoff, Nature 2011, 480, 480; d) A. D. Fesnak, C. H. June, B. L. Levine, Nat. Rev. Cancer 2016, 16, 566.

[2] a) P. C. Tumeh, C. L. Harview, J. H. Yearley, I. P. Shintaku, E. J. Taylor, L. Robert, B. Chmielowski, M. Spasic, G. Henry, V. Ciobanu, A. N. West, M. Carmona, C. Kivork, E. Seja, G. Cherry, A. J. Gutierrez, T. R. Grogan, C. Mateus, G. Tomasic, J. A. Glaspy, R. O. Emerson, H. Robins, R. H. Pierce, D. A. Elashoff, C. Robert, A. Ribas, Nature 2014, 515, 568; b) S. S. Taneja, J. Urol. 2012, 188, 2148; c) O. Hamid, C. Robert, A. Daud, F. S. Hodi, W. J. Hwu, R. Kefford, J. D. Wolchok, P. Hersey, R. W. Joseph, J. S. Weber, R. Dronca, T. C. Gangadhar, A. Patnaik, H. Zarour, A. M. Joshua, K. Gergich, J. Elassaiss-Schaap, A. Algazi, C. Mateus, P. Boasberg, P. C. Tumeh, B. Chmielowski, S. W. Ebbinghaus, X. N. Li, S. P. Kang, A. Ribas, N. Engl. J. Med. 2013, 369, 134; d) C. Robert, A. Ribas, J. D. Wolchok, F. S. Hodi, O. Hamid, R. Kefford, J. S. Weber, A. M. Joshua, W. J. Hwu, T. C. Gangadhar, A. Patnaik, R. Dronca, H. Zarour, R. W. Joseph, P. Boasberg, B. Chmielowski, C. Mateus, M. A. Postow, K. Gergich, J. Elassaiss-Schaap, X. N. Li, R. Iannone, 
S. W. Ebbinghaus, S. P. Kang, A. Daud, Lancet 2014, 384, 1109; e) C. Robert, G. V. Long, B. Brady, C. Dutriaux, M. Maio, L. Mortier, J. C. Hassel, P. Rutkowski, C. McNeil, E. Kalinka-Warzocha, K. J. Savage, M. M. Hernberg, C. Lebbe, J. Charles, C. Mihalcioiu, V. Chiarion-Sileni, C. Mauch, F. Cognetti, A. Arance, H. Schmidt, D. Schadendorf, H. Gogas, L. Lundgren-Eriksson, C. Horak B. Sharkey, I. M. Waxman, V. Atkinson, P. A. Ascierto, N. Engl. J. Med. 2015, 372, 320.

[3] W. Zou, J. D. Wolchok, L. Chen, Sci. Transl. Med. 2016, 8, $328 \mathrm{rv} 4$.

[4] a) C. Robert, J. Schachter, G. V. Long, A. Arance, J. J. Grob, L. Mortier, A. Daud, M. S. Carlino, C. McNeil, M. Lotem, J. Larkin, P. Lorigan, B. Neyns, C. U. Blank, O. Hamid, C. Mateus, R. Shapira-Frommer, M. Kosh, H. Zhou, N. Ibrahim, S. Ebbinghaus, A. Ribas, N. Engl. J. Med. 2015, 372, 2521; b) P. Sharma, J. P. Allison, Science 2015, 348, 56; c) A. Hoos, Nat. Rev. Drug Discovery 2016, 15, 235; d) H. O. Alsaab, S. Sau, R. Alzhrani, K. Tatiparti, K. Bhise, S. K. Kashaw, A. K. lyer, Front. Pharmacol. 2017, 8, 561.

[5] a) L. Riechmann, M. Clark, H. Waldmann, G. Winter, Nature 1988, 332, 323; b) W. W. Gibbs, Sci. Am. 2005, 293, 78.

[6] a) C. M. Hu, L. Zhang, S. Aryal, C. Cheung, R. H. Fang, Proc. Natl. Acad. Sci. USA 2011, 108, 10980; b) C. M. Hu, R. H. Fang, K. C. Wang, B. T. Luk, S. Thamphiwatana, D. Dehaini, P. Nguyen, P. Angsantikul, C. H. Wen, A. V. Kroll, C. Carpenter, M. Ramesh, V. Qu, S. H. Patel, J. Zhu, W. Shi, F. M. Hofman, T. C. Chen, W. Gao, K. Zhang, S. Chien, L. Zhang, Nature 2015, 526, 118; c) Q. Hu, W. Sun, C. Qian, C. Wang, H. N. Bomba, Z. Gu, Adv. Mater. 2015, 27, 7043.

[7] a) S. L. Topalian, J. M. Taube, R. A. Anders, D. M. Pardoll, Nat. Rev. Cancer 2016, 16, 275; b) J. R. Brahmer, S. S. Tykodi, L. Q. Chow, W. J. Hwu, S. L. Topalian, P. Hwu, C. G. Drake, L. H. Camacho, J. Kauh, K. Odunsi, H. C. Pitot, O. Hamid, S. Bhatia, R. Martins, K. Eaton, S. Chen, T. M. Salay, S. Alaparthy, J. F. Grosso, A. J. Korman, S. M. Parker, S. Agrawal, S. M. Goldberg, D. M. Pardoll, A. Gupta, J. M. Wigginton, N. Engl. J. Med. 2012, 366, 2455; c) S. S. Taneja, J. Urol. 2012, 188, 2149.

[8] a) S. Tan, T. Wu, D. Zhang, Z. Zhang, Theranostics 2015, 5, 863; b) P. Vader, E. A. Mol, G. Pasterkamp, R. M. Schiffelers, Adv. Drug Delivery Rev. 2016, 106, 148; c) R. van der Meel, M. H. Fens, P. Vader, W. W. van Solinge, O. Eniola-Adefeso, R. M. Schiffelers, J. Controlled Release 2014, 195, 72; d) B. T. Luk, L. Zhang, J. Controlled Release 2015, 220,600; e) C. Wang, W. Sun, Y. Ye, Q. Hu, H. N. Bomba, Z. Gu, Nat. Biomed. Eng. 2017, 1, 0011; f) M. T. Stephan, J. J. Moon, S. H. Um, A. Bershteyn, D. J. Irvine, Nat. Med. 2010, 16, 1035; g) H. Zhou, Z. Fan, P. K. Lemons, H. Cheng, Theranostics 2016, 6, 1012
[9] a) L. Gu, D. J. Mooney, Nat. Rev. Cancer 2016, 16, 56; b) S. T. Koshy, D. J. Mooney, Curr. Opin. Biotechnol. 2016, 40, 1; c) M. S. Goldberg, Cell 2015, 161, 201; d) L. Jeanbart, M. A. Swartz, Proc. Natl. Acad. Sci. USA 2015, 112, 14467; e) L. M. Kranz, M. Diken, H. Haas, S. Kreiter, C. Loquai, K. C. Reuter, M. Meng, D. Fritz, F. Vascotto, H. Hefesha, C. Grunwitz, M. Vormehr, Y. Husemann, A. Selmi, A. N. Kuhn, J. Buck, E. Derhovanessian, R. Rae, S. Attig, J. Diekmann, R. A. Jabulowsky, S. Heesch, J. Hassel, P. Langguth, S. Grabbe, C. Huber, O. Tureci, U. Sahin, Nature 2016, 534, 396; f) G. M. Lynn, R. Laga, P. A. Darrah, A. S. Ishizuka, A. J. Balaci, A. E. Dulcey, M. Pechar, R. Pola, M. Y. Gerner, A. Yamamoto, C. R. Buechler, K. M. Quinn, M. G. Smelkinson, O. Vanek, R. Cawood, T. Hills, O. Vasalatiy, K. Kastenmuller, J. R. Francica, L. Stutts, J. K. Tom, K. A. Ryu, A. P. Esser-Kahn, T. Etrych, K. D. Fisher, L. W. Seymour, R. A. Seder, Nat. Biotechnol. 2015, 33, 1201; g) H. Liu, K. D. Moynihan, Y. Zheng, G. L. Szeto, A. V. Li, B. Huang, D. S. Van Egeren, C. Park, D. J. Irvine, Nature 2014, 507, 519; h) J. Park, S. H. Wrzesinski, E. Stern, M. Look, J. Criscione, R. Ragheb, S. M. Jay, S. L. Demento, A. Agawu, P. L. Limon, Nat. Mater. 2012, 11, 895; i) T. R. Fadel, F. A. Sharp, N. Vudattu, R. Ragheb, J. Garyu, D. Kim, E. P. Hong, N. Li, G. L. Haller, L. D. Pfefferle, S. Justesen, K. C. Herold, T. M. Fahmy, Nat. Nanotechnol. 2014, 9, 639; j) J. Kim, W. A. Li, Y. Choi, S. A. Lewin, C. S. Verbeke, G. Dranoff, D. J. Mooney, Nat. Biotechnol. 2015, 33, 64; k) S. B. Stephan, A. M. Taber, I. Jileaeva, E. P. Pegues, C. L. Sentman, M. T. Stephan, Nat. Biotechnol. 2015, 33, 97; I) R. Kuai, L. J. Ochyl, K. S. Bahjat, A. Schwendeman, J. J. Moon, Nat. Mater. 2017, 16, 489.

[10] D. H. Munn, A. L. Mellor, J. Clin. Invest. 2007, 117, 1147.

[11] G. Frumento, R. Rotondo, M. Tonetti, G. Damonte, U. Benatti, G. B. Ferrara, J. Exp. Med. 2002, 196, 459.

[12] a) M. Xie, H. Ye, H. Wang, G. Charpin-El Hamri, C. Lormeau, P. Saxena, J. Stelling, M. Fussenegger, Science 2016, 354, 1296; b) P. Zhang, Y. Chen, Y. Zeng, C. Shen, R. Li, Z. Guo, S. Li, Q. Zheng, C. Chu, Z. Wang, Z. Zheng, R. Tian, S. Ge, X. Zhang, N. S. Xia, G. Liu, X. Chen, Proc. Natl. Acad. Sci. USA 2015, 112, E6129.

[13] a) R. H. Fang, C. M. Hu, B. T. Luk, W. Gao, J. A. Copp, Y. Tai, D. E. O'Connor, L. Zhang, Nano Lett. 2014, 14, 2181; b) S. C. Jang, O. Y. Kim, C. M. Yoon, D. S. Choi, T. Y. Roh, J. Park, J. Nilsson, J. Lotvall, Y. K. Kim, Y. S. Gho, ACS Nano 2013, 7, 7698.

[14] E. J. Wherry, Nat. Immunol. 2011, 12, 492.

[15] E. J. Wherry, S. J. Ha, S. M. Kaech, W. N. Haining, S. Sarkar, V. Kalia, S. Subramaniam, J. N. Blattman, D. L. Barber, R. Ahmed, Immunity 2007, 27, 670 .

[16] L. C. Wu, A. A. Zarrin, Nat. Rev. Immunol. 2014, 14, 247.

[17] C. Wang, Y. Ye, Q. Hu, A. Bellotti, Z. Gu, Adv. Mater. 2017, 29, 1606036. 